\title{
Krankheitsbezogene Ängste und Depressivität bei COPD-Patienten
}

\author{
COPD-Specific Anxiety and Depressive Symptoms in COPD Patients
}

Autoren

Institute
N. Zöckler ${ }^{1}$, W. Rief ${ }^{1}$, K. Kühl', K. Kenn ${ }^{2}$

${ }^{1}$ Philipps-Universität Marburg, AG Klinische Psychologie und Psychotherapie

${ }^{2}$ Schön Klinik Berchtesgadener Land $\begin{array}{lll}\text { eingereicht } & \text { 26. 10. } 2011\end{array}$ akzeptiert nach Revision 23. 12.2011

Bibliografie

DOI http://dx.doi.org/

10.1055/s-0031-1291636

Online-Publikation: 23.2.2012

Pneumologie 2012; 66: 290-296

(c) Georg Thieme Verlag KG

Stuttgart · New York

ISSN 0934-8387

\section{Korrespondenzadresse}

\section{Natalie Zöckler}

Philipps-Universität Marburg

AG Klinische Psychologie und

Psychotherapie

Gutenbergstraße 18

35032 Marburg

Natalie.Zoeckler@staff.uni-

marburg.de

\section{Zusammenfassung \\ $\nabla$}

Ziel der vorliegenden Studie ist die Analyse von krankheitsbezogenen Ängsten im Zusammenhang mit Depressivität bei COPD-Patienten. Sowohl Angst als auch Depressivität sind ein häufiges komorbides Phänomen bei COPD. Beides kann einen erheblichen Einfluss auf den Verlauf und das Erleben der Erkrankung haben. Bislang wurden in Studien lediglich allgemeine - nicht aber COPD-assoziierte - Ängste erfasst. An der Studie nahmen insgesamt 96 COPD-Patienten (GOLD III/ IV) teil. Die MANOVA ergab, dass COPD-Patienten mit auffälligen Depressivitätswerten stärkere krankheitsbezogene Ängste berichten als Patienten mit unauffälligen Depressivitätswerten. Sie leiden unter größerer Angst vor sozialer Ausgrenzung, Angst vor Dyspnoe, Angst vor körperlicher Aktivität und Progredienzangst. Es ergaben sich keine Unterschiede zwischen den beiden Vergleichsgruppen in den medizinisch-objektiven Parametern ( $\mathrm{FEV}_{1} \%$ des Solls; 6-Minuten-Gehtest). Insgesamt belegt unsere Studie die hohe Relevanz der Erfassung krankheitsspezifischer Ängste bei COPD-Patienten im Zusammenhang mit Depressivität. Die rechtzeitige Identifikation und angemessene Behandlung von COPD-bezogenen Ängsten und Depressivität hat eine große Bedeutung für den Krankheitsverlauf.

\section{Theoretischer Hintergrund}

$\nabla$

Die chronisch-obstruktive Lungenerkrankung (COPD) zählt zu den progredient und irreversibel verlaufenden Erkrankungen mit stetig steigenden Prävalenzraten [1]. In Deutschland leiden derzeit etwa 5 Millionen Menschen unter COPD, wobei die Prävalenz bei über 40-Jährigen $8,7 \%$ und bei über 70 -Jährigen $19 \%$ beträgt [2]. Weltweit ist die COPD derzeit die vierthäufigste Todesursache und es wird erwartet, dass die Mortalität bis zum Jahre 2030 an die dritte Stelle der Statistik für

\section{Abstract \\ $\nabla$}

The aim of the present study was to analyze the associations between COPD-specific anxiety and depressive symptoms in COPD patients. Anxiety, as well as depression, is a prevalent comorbid complication in COPD. Either can have a negative impact on patients' experience and the course of disease. So far, assessment in studies has exclusively focused on anxiety in general and not on disease-related fears. The present study comprised a sample of ninety-six patients suffering from COPD (GOLD III/IV). Results of the MANOVA showed higher COPD-specific anxiety in patients with depressive symptoms than in patients without depressive symptoms. Depressive patients showed a higher fear of social isolation, dyspnea related fear, fear of movement and fear of progression of disease. There were no differences between the two groups with regard to functional parameters ( $\mathrm{FEV}_{1} \%$ pred.; 6-minute-walk-test). Overall, our study shows the necessity of the assessment of COPD-specific fears related to depression to guarantee an adequate treatment.

Todesursachen vorrücken wird [1]. Zu den charakteristischen Symptomen zählen Husten, Dyspnoe, eingeschränkte Lungenfunktion und Belastungsintoleranz [3].

COPD-Patienten sind aufgrund ihrer Erkrankung zunehmend in sämtlichen Lebensbereichen massiv eingeschränkt und berichten im Krankheitsverlauf über eine immer weiter sinkende gesundheitsbezogene Lebensqualität [4]. Zusätzliche Beeinträchtigungen entstehen durch psychische Symptome wie Depressivität und Ängstlichkeit [5-9]. Es ergeben sich Schwierigkeiten in der Di- 
agnostik, da häufig unklar ist, ob die beschriebene Depressions-/ Angstsymptomatik Ausdruck der körperlichen Erkrankung ist oder eine eigenständige psychische Störung widerspiegelt. Um komorbide psychische Störungen bei Patienten mit somatischen Erkrankungen zu erkennen, wird für die klinische Praxis ein routinemäßiges Screening psychischer Belastungen empfohlen [10]. Im Anschluss daran wird eine psychodiagnostische Untersuchung positiv gescreenter Patienten vorgeschlagen, die als Grundlage zur Indikationsstellung dient. Dieses Vorgehen kann zu einem frühzeitigen Erkennen psychischer Komorbidität beitragen und Chronifizierungsprozessen entgegenwirken [10].

Definitorisch werden im Folgenden Depression, Depressivität, krankheitsspezifische und allgemeine Ängste voneinander abgegrenzt. Unter Depression versteht man eine psychiatrisch diagnostizierte Erkrankung, bei der die Einschränkung des emotionalen Erlebens sowie verminderter Antrieb und Aktivität im Vordergrund steht $[11,12]$. Mithilfe von Selbstbeurteilungsverfahren erfasst man die von den Patienten selbst eingeschätzte Depressivität. Von Depressivität spricht man, wenn eher leichte Symptome einer psychischen Auffälligkeit vorliegen (im Sinne von Vorboten einer psychischen Erkrankung). Sowohl COPD-spezifische als auch krankheitsbezogene Ängste bedeuten nachvollziehbare, realistische Ängste in Bezug auf die körperliche Symptomatik (Exazerbation, Dyspnoe) und die daraus resultierenden Konsequenzen für die Zukunft (Angst vor dem Fortschreiten der Erkrankung). Angststörungen hingegen werden oftmals geprägt von dem Gefühl der Furcht vor bestimmten Situationen, wobei die Aktivität und der Antrieb, abgesehen von angstauslösenden Situationen, häufig nicht beeinträchtigt sind [12]. Das Konstrukt der COPD-spezifischen Ängste weist Überschneidungen mit dem Konstrukt von Angststörungen auf. Ein konzeptioneller Unterschied besteht jedoch darin, dass bei der Erfassung COPD-assoziierter Ängste die Aufmerksamkeit insbesondere auf atemnotbezogene Befürchtungen, bei der Panikstörung z.B. eher auf allgemeine, unterschiedliche Körperempfindungen gerichtet ist [13]. Zudem sind die COPD-bezogenen Befürchtungen auf Folgen für die Zukunft ausgerichtet und erfassen somit ein breiteres Spektrum als die Konstrukte spezifischer Angststörungen.

Angst gehört neben Depressivität zu den häufigsten psychischen Komorbiditäten bei COPD $[14,15,16]$. Erschwert wird der COPDVerlauf durch krankheitsspezifische Ängste, die als komorbides Syndrom auftreten können. Bislang wurden in Studien nur Fragebögen zur Erfassung allgemeiner, nicht aber COPD-assoziierter Ängste eingesetzt. Bei anderen schweren, chronisch verlaufenden Erkrankungen, wie koronaren Herzerkrankungen, Diabetes mellitus oder Tumorerkrankungen, wurde die Bedeutung krankheitsbezogener Ängste bereits deutlich [17,18]. Kühl et al. [19] entwickelten in ihrer Studie ein Instrument zur Erfassung COPD-spezifischer Ängste. Als relevante Bereiche für COPD-Patienten stellten sich Progredienzangst, Angst vor sozialer Ausgrenzung, Angst vor Dyspnoe und die Angst vor körperlicher Aktivität heraus [18-21].

Gemäß einer Meta-Analyse weisen 36\% der COPD-Patienten erhöhte Angstwerte auf [14]. In Selbstbeurteilungsfragebögen berichten $13 \%$ bis $96 \%$ von Ängstlichkeit [22, 23]. Bei COPD-Patienten ist Angst assoziiert mit einer größeren subjektiven Beeinträchtigung [24,25], verstärkt wahrgenommener Atemnot [26], verminderter körperlicher Leistungsfähigkeit [24], einer höheren Anzahl an Arztbesuchen [27] und Krankenhausaufnahmen [14, 28,29]. Außerdem korreliert die Höhe des Angstlevels mit der Anzahl negativer Kognitionen [30]. Dyspnoe stellt als Leitsymptom der COPD eine potenzielle Angstquelle dar und steht in einem engen Zusammenhang mit Angst- und Panikstörungen $[29,31,32]$. Betroffene mit ausgeprägten Ängsten weisen ein schlechteres Symptommanagement auf, verfügen über weniger Copingstrategien und haben eine geringere Selbstwirksamkeitserwartung [33]. Ängstliche COPD-Patienten nehmen atembezogene Symptome intensiver wahr als nicht-ängstliche Patienten $[20,26,34]$. Aufgrund der erhöhten Sensitivität vermeiden Patienten aus Angst vor Atemnot körperliche Aktivitäten, was wiederum im Sinne eines Teufelskreises die allgemeine körperliche Leistungsfähigkeit reduziert. Häufig zeigen sich im psychosozialen Bereich, vor allem in Partnerschaften, ausgeprägte Beeinträchtigungen, da bspw. Freizeit- und soziale Aktivitäten seltener gemeinsam ausgeübt werden können [35,36].

Laut einer Metaanalyse weisen 40\% der COPD-Patienten erhöhte Depressivitätswerte auf [14]. Ein Review beschreibt Prävalenzraten von $2 \%$ bis $57 \%$ [37]. Depression geht einher mit einer schlechteren Überlebensprognose, längeren Krankenhausliegezeiten, einem erhöhten Wiederaufnahmerisiko in klinische Einrichtungen und gilt als Risikofaktor für das Scheitern einer pneumologischen Rehamaßnahme $[28,38,39]$. Zahlreiche Untersuchungen belegen Zusammenhänge zwischen Depressivität und einer verminderten körperlichen Leistungsfähigkeit [27,40,41]. Sowohl Angst als auch Depressivität spielen bei schweren, chronischen Krankheiten eine bedeutsame Rolle, sind aber unterschiedlich [15]. Es ist wichtig, nicht nur allgemeine Ängstlichkeit, sondern ebenfalls COPD-spezifische Ängste in Relation zu Depressivität zu betrachten. Die vorliegende Studie analysiert erstmalig diesen Aspekt, was in bisherigen Studien keine Berücksichtigung fand.

Die folgenden Fragen sollen im Einzelnen beantwortet werden:

1. Unterscheiden sich COPD-Patienten mit auffälligen Depressivitätswerten in ihren krankheitsspezifischen Ängsten von COPD-Patienten mit unauffälligen Depressivitätswerten?

2. Gibt es einen Unterschied zwischen COPD-Patienten mit auffälligen Depressivitätswerten und COPD-Patienten mit unauffälligen Depressivitätswerten in den angstbezogenen Kognitionen und der Angst vor körperlichen Symptomen?

3. Unterscheiden sich COPD-Patienten mit auffälligen Depressivitätswerten in den medizinisch-objektiven Parametern von COPD-Patienten mit unauffälligen Depressivitätswerten?

\section{Methoden \\ $\nabla$}

Untersuchungsablauf und Stichprobe

Die Datenerhebung fand im Rahmen einer Studie zur Untersuchung von psychosozialen Bewältigungsmechanismen bei Patienten mit COPD über einen Zeitraum von neun Monaten im Jahre 2008 in der Schön Klinik Berchtesgadener Land statt. Es wurden konsekutiv alle mit der Studienteilnahme einverstandenen COPD-Patienten (GOLD-Stadien III und IV) eingeschlossen. Dabei handelte es sich um Betroffene mit oder ohne Sauerstofflangzeittherapie, mit oder ohne nicht-invasive Beatmung als auch mit oder ohne Indikation zur Lungentransplantation. Ausgeschlossen waren Patienten mit akutem Koronarsyndrom, Herzinsuffizienz und malignen Tumorerkrankungen. Schwere Exazerbationen der COPD im Verlauf galten als Abbruchkriterium. Nach der Aufklärung über die Studie durch den Untersuchungsleiter unterschrieben alle Patienten zu Beginn einen „,informed consent“. Die Teilnehmer wurden einen Tag nach Aufnahme und einen Tag vor Abreise psychologisch (Fragebogenerhebung, halbstrukturierte Interviews) und medizinisch (Lungenfunktionsanalyse, 6-Minu- 


\begin{tabular}{|c|c|c|c|c|}
\hline & $\begin{array}{l}\text { Gesamt } \\
n=96\end{array}$ & $\begin{array}{l}\text { HADS-D }(0-7) \\
n=51\end{array}$ & $\begin{array}{l}\text { HADS-D (8-21) } \\
n=45\end{array}$ & Teststatistik $^{a}$ \\
\hline \multicolumn{5}{|l|}{ Geschlecht } \\
\hline Frauen & $55(57,3)$ & $24(47,1)$ & $31(68,9)$ & \\
\hline Männer & $41(42,7)$ & $27(52,9)$ & $14(31,1)$ & $\mathrm{Chi}^{2}=4,656^{*}$ \\
\hline \multicolumn{5}{|l|}{ COPD-Stadium } \\
\hline Schweregrad III & $26(27,1)$ & $16(31,4)$ & $10(22,2)$ & \\
\hline Schweregrad IV & $70(72,9)$ & $35(68,6)$ & $35(77,8)$ & $C h i^{2}=1,014$ \\
\hline Alter (Jahre) M (SD) & $62,21(8,27)$ & $62,21(8,27)$ & $59,89(9,30)$ & $t=1,297$ \\
\hline BMI M (SD) & $24,38(5,41)$ & $24,50(5,26)$ & $24,24(5,63)$ & $t=0,230$ \\
\hline \multicolumn{5}{|l|}{ Familienstand (\%) } \\
\hline verheiratet/zusammenlebend & $71(74,0)$ & $37(72,5)$ & $34(75,6)$ & \\
\hline geschieden und ledig & $25(26,0)$ & $14(27,5)$ & $11(24,4)$ & $\mathrm{Chi}^{2}=1,218$ \\
\hline
\end{tabular}

Tab. 1 Stichprobenbeschreibung; Vergleich soziodemographischer Merkmale und medizinischer Kennwerte zwischen Patienten mit unauffälligen Depressivitätswerten (HADS-D; 0 - 7) und Patienten mit auffälligen Depressivitätswerten (HADS-D; 8-21).

HADS-D = Hospital Anxiety and Depression Scale. Mittelwert \pm Standardabweichungen, absolute (n) und relative (\%) Häufigkeitsangaben; a t-tests für Alter und BMI; Chi-Quadrat-Tests für Geschlecht, COPD-Stadium und Familienstand; * $p<0,05$.

ten-Gehtest) untersucht. Zehn Patienten mussten aufgrund akuter Exazerbation ausgeschlossen werden bzw. lehnten eine Teilnahme ab. Wie aus $\bullet$ Tab. 1 ersichtlich, gingen 96 vollständige Datensätze in die Baselineerhebung ein.

\section{Messinstrumente}

Hospital Anxiety and Depression Scale (HADS)

Die Hospital Anxiety and Depression Scale [42] stellt ein Selbstbeurteilungsverfahren zur Erfassung der Ausprägung von Angst und Depressivität während der vergangenen Woche bei Patienten mit körperlichen Erkrankungen dar. Das Verfahren kann als Screening und zur Verlaufsbeurteilung eingesetzt werden. Es erfasst eher leichte Symptome einer psychischen Auffälligkeit im Sinne von Vorboten einer möglichen psychischen Erkrankung. Die Subskala Depressivität (HADS-D) besteht aus sieben Items (Itemwerte 0 - 3 ). Subskalenwerte $\geq 8$ weisen auf erhöhte, auffällige Depressivitätswerte hin. Die Depressivitätsskala erfragt Aspekte von Interessenverlust und Verlust der Freude. Itemauswahl und -formulierung berücksichtigen die spezifischen Anforderungen eines durch körperliche Krankheit bestimmten Settings.

\section{COPD-Angst-Fragebogen (CAF)}

Der COPD-Angst-Fragebogen [19] erfasst krankheitsbezogene Ängste, die durch COPD hervorgerufen werden. Der Fragebogen besteht aus vier Subskalen. Die Skala „Progredienzangst“ (PA) enthält Items, welche die Angst vor dem Fortschreiten der Erkrankung erfassen. Die Skala „Angst vor sozialer Ausgrenzung“ (ASA) beschreibt Befürchtungen hinsichtlich der Auswirkungen auf soziale Beziehungen und Aktivitäten aufgrund der COPD. „Angst vor Dyspnoe“ (AD) beschreibt Ängste und Befürchtungen im Zusammenhang mit Atemnot. Die Subskala „Angst vor körperlicher Aktivität“ (AKA) erfragt die Vermeidung von körperlicher Anstrengung. Des Weiteren beschreiben zwei Zusatzskalen die „Angst bezüglich Partnerschaft“ und „Angst bezüglich Langzeitsauerstofftherapie“. Insgesamt enthält der Fragebogen 27 Items (zusätzlich 8 Items für die zwei fakultativen Skalen). Das Antwortformat reicht hierbei mit einer fünfstufigen Skala von „nie“ (0) bis „immer“ (4). Das Instrument verfügt über eine gute Reliabilität und zufriedenstellende Validität.

\section{Fragebogen zu angstbezogenen Kognitionen (ACQ)}

Der Fragebogen zu angstbezogenen Kognitionen (Agoraphobic Cognitions Questionnaire [43]) erfasst mit Hilfe von 14 Items das Ausmaß angstbezogener Kognitionen bei Patienten. Zur Beantwortung gibt der Patient auf einer Fünf-Punkte-Skala an, wie häufig jede aufgeführte Kognition bei ihm auftritt, wenn er gerade nervös oder ängstlich ist. Die Skala reicht hierbei von „Der Gedanke kommt nie vor“ (1) bis „Der Gedanke kommt immer vor" (5).

Fragebogen zur Angst vor körperlichen Symptomen (BSQ) Der Fragebogen zur Angst vor körperlichen Symptomen (Body Sensations Questionnaire [43]) erfasst, wie stark die Angst vor körperlichen Symptomen ausgeprägt ist. Die Patienten werden aufgefordert, jeweils auf einer Fünf-Punkte-Skala anzugeben, in welchem Ausmaß das betreffende körperliche Symptom ihnen Angst oder Sorgen macht. Die Skala reicht hierbei von „gar nicht beunruhigt oder ängstlich“ (1) bis „extrem ängstlich“ (5). Das Instrument besteht insgesamt aus 17 Items.

\section{Medizinische Parameter}

Als objektive Parameter wurde eine Lungenfunktionsdiagnostik gemäß der Richtlinien der American Thoracic Society und der 6Minuten-Gehtest [44] durchgeführt. Die Einsekundenkapazität $\mathrm{FEV}_{1}$ (Forced Expiratory Volume in 1 second) ist ein dynamischer, zeitabhängiger Messparameter in der Lungenfunktionsdiagnostik. Der Wert dient u.a. der Stadienzuordnung nach GOLD bei COPD.

Der 6-Minuten-Gehtest wird eingesetzt, um die funktionelle Kapazität und das Ausmaß der Belastbarkeit von Patienten mit COPD zu bestimmen. Der Patient läuft über den Zeitraum von sechs Minuten auf ebener Erde, wobei er das Tempo selbst vorgibt mit dem Ziel, der eigenen Leistung entsprechend möglichst weit zu laufen. Pausen sind dabei erlaubt. Es wird die von dem Patienten zurückgelegte Wegstrecke in Metern gemessen.

\section{Statistische Auswertung}

Die statistische Auswertung wurde mit SPSS für Windows Version 18.0 vorgenommen. Zunächst wurden die statistischen Verteilungsformen mit dem Kolmogorov-Smirnov-Test überprüft. Für die Vergleiche zwischen der Gruppe mit unauffälligen Depressivitätswerten (HADS-D; 0-7) und der Gruppe mit auffälligen Depressivitätswerten (HADS-D; 8-21) bezüglich der soziodemographischen Kennwerte wurden t-Tests für unabhängige Stichproben verwendet, bei nicht intervallskalierten Daten der Chi-Quadrat-Test. Zur Ermittlung von Unterschieden in Abhängigkeit von der Untersuchungsgruppe wurden multivariate Varianzanalysen (MANOVA) berechnet. Um differenzierter klären zu können, in welchen Variablen sich die beiden Gruppen unterscheiden, wurden anschließend univariate Varianzanalysen berechnet. Das Signifikanzniveau wurde für alle Analysen auf 0,05 festgelegt. 


\section{Ergebnisse}

\section{Stichprobe}

Wie aus $\bullet$ Tab. 1 ersichtlich, nahmen an der Untersuchung insgesamt $N=96$ (55 Frauen, 57,3\%) COPD-Patienten teil. Das Durchschnittsalter der Studienteilnehmer betrug 61,2 (SD=8,8) Jahre. Bezüglich des Familienstandes gaben $72,9 \%$ der Befragten an, verheiratet zu sein oder in einer Partnerschaft zu leben, 26,0\% waren geschieden und ledig. Zum Zeitpunkt der Erhebung litten 27,1\% der Patienten unter einer schweren COPD (Stadium III), $72,9 \%$ unter einer sehr schweren COPD (Stadium IV). Der durchschnittliche BMI der Gesamtstichprobe betrug 24,38 ( $S D=5,41$ ), der $\mathrm{FEV}_{1} \%$ vom Soll betrug 36,7 (SD=11,65), die 6-Minuten-Gehstrecke belief sich im Mittel auf 293 Meter (SD=144). 81,2\% der Patienten erhielten eine Langzeitsauerstofftherapie, 27,1\% erhielten eine nächtliche nicht-invasive Beatmung (NIV) und $28,1 \%$ waren für eine Lungentransplantation vorgesehen.

\section{Stichprobenbeschreibung}

Die Ergebnisse der Vergleiche soziodemographischer Merkmale und medizinischer Kennwerte zwischen der Gruppe mit unauffälligen Depressivitätswerten (HADS-D; 0-7) und der Gruppe mit auffälligen Depressivitätswerten (HADS-D; 8-21) sind in - Tab. 1 dargestellt. Insgesamt zeigte sich hierbei ein signifikanter Gruppenunterschied bezüglich des Geschlechts $\left(\chi^{2}(1)=4,656\right.$, $\mathrm{p}<0,05)$. Es fanden sich keine signifikanten Gruppenunterschiede bezüglich der Kennwerte COPD-Stadium $\left(\chi^{2}(1)=1,014, p>\right.$ $0,05)$, Alter $(t(94)=1,297, \mathrm{p}>0,05)$, BMI $(t(94)=0,479, \mathrm{p}>0,05)$ und Familienstand $\left(x^{2}(1)=1,218, p>0,05\right)$.

\section{COPD-spezifische Angstbereiche}

Bezüglich der COPD-spezifischen Ängste wurden die Patienten mit unauffälligen Depressivitätswerten (HADS-D; 0-7) mit den Patienten mit auffälligen Depressivitätswerten (HADS-D; 8-21) verglichen. In der multivariaten Varianzanalyse zeigte sich ein signifikanter Haupteffekt der COPD-bezogenen Angstbereiche $(F$ $\left.(4,91)=11,799, \mathrm{p} \leq 0,001, \eta^{2}=.342\right)$. Anschließende univariate Varianzanalysen ergaben, dass sich die beiden Gruppen in allen vier Angstbereichen (Progredienzangst, Angst vor sozialer Ausgrenzung, Angst vor Dyspnoe und Angst vor körperlicher Aktivität) signifikant voneinander unterscheiden. Es wurde deutlich, dass die Patienten mit auffälligen Depressivitätswerten signifikant mehr Progredienzangst $\left(F(1,94)=26,276, \mathrm{p} \leq 0,001, \eta^{2}=.218\right)$, Angst vor sozialer Ausgrenzung $\left(F(1,94)=37,083, \mathrm{p} \leq 0,001, \eta^{2}=.283\right)$, Angst vor Dyspnoe $\left(F(1,94)=14,152, \mathrm{p} \leq 0,001, \eta^{2}=.131\right)$ und Angst vor körperlicher Aktivität $\left(F(1,94)=5,002, \mathrm{p}=0,028, \eta^{2}=\right.$ .051) als Patienten mit unauffälligen Depressivitätswerten berichten. Ergebnisse der multivariaten Varianzanalyse sowie Mittelwerte und Standardabweichungen der Gruppen hinsichtlich der COPD-spezifischen Angstbereiche sind in $\bullet$ Tab. 2 dargestellt.

Des Weiteren wurde mittels einer multivariaten Varianzanalyse untersucht, ob sich die Gruppe mit unauffälligen Depressivitätswerten (HADS-D; 0 - 7) von der Gruppe mit auffälligen Depressivitätswerten (HADS-D; 8-21) in den Variablen angstbezogene Kognitionen (ACQ), Angst vor körperlichen Symptomen (BSQ), $\mathrm{FEV}_{1} \%$ des Solls und 6-Minuten-Gehstrecke unterscheiden. Es ergab sich ein signifikanter Haupteffekt der Gruppen $(F(5,90)=$ $\left.8,686, p \leq 0,001, \eta^{2}=.325\right)$. Anschließende univariate Varianzanalysen ergaben, dass sich die beiden Gruppen lediglich in der Variablen BSQ signifikant unterscheiden. Die Patienten mit auffälligen Depressivitätswerten berichten im BSQ signifikant mehr Angst vor körperlichen Symptomen $(F(1,94)=4,406, p=0,038$, $\eta^{2}=.045$ ). Es konnte kein Unterschied zwischen den beiden Gruppen bezüglich der objektiv erhobenen Parameter $\mathrm{FEV}_{1} \%$ des Solls und 6-Minuten-Gehstrecke bestätigt werden (vgl. @ Tab.3).

Tab. 2 Ergebnisse der Multivariaten Varianzanalyse (MANOVA) für die COPD-spezifischen Angstbereiche mit dem Faktor Gruppe (Patienten mit unauffälligen Depressivitätswerten (HADS-D; 0 - 7) und Patienten mit auffälligen Depressivitätswerten (HADS-D; 8 - 21).

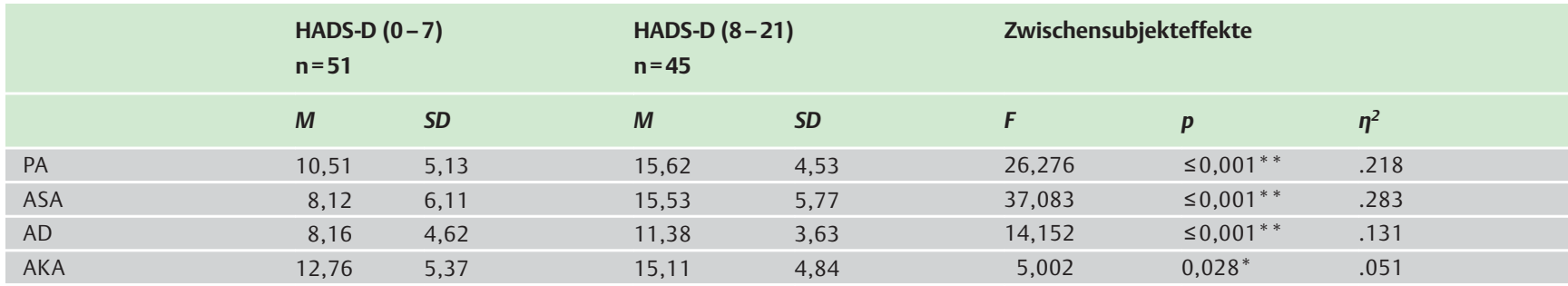

HADS-D = Hospital Anxiety and Depression Scale. $M=$ Mittelwert, $S D=$ Standardabweichung. $P A=$ Progredienzangst, $A S A=$ Angst vor sozialer Ausgrenzung, $A D=$ Angst vor Dyspnoe, AKA = Angst vor körperlicher Aktivität. ${ }^{*} p<0,05,{ }^{*}{ }^{*} p \leq 0,001 ; \eta^{2}=$ Effektstärkemaß Eta-Quadrat ( $\eta^{2} \geq .01$ kleiner Effekt, $\eta^{2} \geq .058$ mittlerer Effekt, $\eta^{2} \geq .138$ großer Effekt).

Tab. 3 Ergebnisse der Multivariaten Varianzanalyse (MANOVA) für den ACQ-Summenwert, BSQ-Summenwert, FEV-Wert und Gehstrecke mit dem Faktor Gruppe (Patienten mit unauffälligen Depressivitätswerten (HADS-D; 0 - 7) und Patienten mit auffälligen Depressivitätswerten (HADS-D; 8 - 21).

\begin{tabular}{|c|c|c|c|c|c|c|c|}
\hline & \multicolumn{2}{|c|}{$\begin{array}{l}\text { HADS-D }(0-7) \\
n=51\end{array}$} & \multicolumn{2}{|c|}{$\begin{array}{l}\text { HADS-D }(8-21) \\
n=45\end{array}$} & \multicolumn{3}{|c|}{ Zwischensubjekteffekte } \\
\hline & $M$ & $S D$ & $M$ & $S D$ & $F$ & $p$ & $\eta^{2}$ \\
\hline ACQ & 19,45 & 5,78 & 20,53 & 4,63 & 1,006 & 0,319 & .011 \\
\hline BSQ & 32,49 & 9,65 & 37,27 & 11,32 & 4,406 & $0,038^{*}$ & .045 \\
\hline $\mathrm{FEV}_{1} \%$ des Solls & 32,55 & 9,49 & 34,60 & 15,30 & 0,637 & 0,427 & .007 \\
\hline Gehstrecke & 303,68 & 118,54 & 283,71 & 155,06 & 0,509 & 0,477 & .005 \\
\hline
\end{tabular}

$\mathrm{M}=$ Mittelwert, $\mathrm{SD}=$ Standardabweichung; Wilks' lambda $=.688, p \leq 0,001$. HADS-D = Hospital Anxiety and Depression Scale, ACQ= Agoraphobic Cognitions Questionnaire, $\mathrm{BSQ}=$ Body Sensations Questionnaire, $\mathrm{FEV}_{1} \%$ des Solls $=$ Einsekundenkapazität in Prozent des Solls, Gehstrecke $=6$-Min.-Gehstrecke in Metern. ${ }^{*} p<0,05$; $\eta^{2}=$ Effektstärkemaß Eta-Quadrat ( $\eta^{2} \geq .01$ kleiner Effekt, $\eta^{2} \geq .058$ mittlerer Effekt, $\eta^{2} \geq .138$ großer Effekt). 


\section{Diskussion}

$\nabla$

In der vorliegenden Studie erfolgte erstmalig, im Gegensatz zu den eingangs erwähnten Arbeiten, ein Vergleich zwischen COPD-Patienten mit auffälligen Depressivitätswerten und unauffälligen Depressivitätswerten hinsichtlich ihrer krankheitsspezifischen Ängste, angstbezogenen Kognitionen, Angst vor körperlichen Symptomen und medizinisch-objektiven Parametern.

Insgesamt 43,2\% der Untersuchten berichten in dem Selbstbeurteilungsverfahren von Depressivität, was im Einklang mit den Ergebnissen anderer Studien steht [14,37]. Zudem leiden Betroffene mit auffälligen Depressivitätswerten unter signifikant stärkeren COPD-spezifischen Ängsten als Betroffene mit unauffälligen Depressivitätswerten. Befunde bei anderen schweren körperlichen Erkrankungen ergaben, dass bei komorbider Depression krankheitsspezifische Ängste einen substantiellen Belastungsfaktor darstellen $[18,45]$, wobei diese Ergebnisse nicht direkt vergleichbar sind mit der hier durchgeführten Studie, da hierbei lediglich Depressivität erfasst wurde. Das gefundene Resultat könnte jedoch ein Hinweis darauf sein, dass Depressivität COPDspezifische Ängste verstärkt. Offen dabei bleibt, ob als primäre Ursache Depressivität COPD-bezogene Befürchtungen mit sich bringt oder ob die vermehrte Wahrnehmung von krankheitsspezifischen Ängsten die Entwicklung einer negativen Stimmungslage fördert [46]. Alarmierend ist jedoch die Tatsache, dass Patienten mit auffälligen Depressivitätsausprägungen zusätzlich unter massiven, COPD-spezifischen Ängsten leiden. Somit ist Depressivität ein Indikator für einen veränderten Umgang mit COPD-Symptomen und höherer Angst vor Krankheitsfolgen.

Betrachtet man die einzelnen COPD-spezifischen Bereiche genauer, lässt sich feststellen, dass sich die verglichenen Patientengruppen hinsichtlich ihres Ausmaßes an Progredienzangst, Angst vor sozialer Ausgrenzung, Dyspnoeangst und Angst vor körperlicher Aktivität unterscheiden. Insbesondere hohe Ausprägungen in den Subskalen „Angst vor Dyspnoe“ und „Angst vor körperlicher Aktivität“ stehen Zielen pneumologischer Rehamaßnahmen im Wege [47]. Laut der Leitlinie der deutschen Atemwegsliga stellt die Erhöhung der körperlichen Leistungsfähigkeit bei COPD ein entscheidendes Rehabilitationsziel dar [47], da dadurch eine Linderung der Belastungsdyspnoe und zugleich eine Steigerung der generellen Belastbarkeit von Patienten erreicht werden kann [48]. Insbesondere im Kontext der Rehabilitationsarbeit wird deshalb darauf geachtet, dass Patienten sich aktiv mit ihren Ängsten vor Überforderung auseinandersetzen. Verfügen Patienten über ausgeprägte Angst vor körperlicher Anstrengung, so ist davon auszugehen, dass das Rehabilitationsziel gefährdet ist. Aus dem Bereich der chronischen Schmerzforschung ist bekannt, dass Patienten im Sinne eines „Furchtvermeidungsmodells“ aus Angst vor Schmerzen ihren Bewegungsradius einschränken [49]. Übertragen auf die COPD würde dies bedeuten, dass sich bei Betroffenen aus Angst vor Atemnot über Konditionierungsprozesse eine Furcht vor Belastungsdyspnoe entwickelt. Infolge des Schonund Vermeidungsverhaltens, ähnlich wie bereits für Patienten mit chronischem Schmerz belegt [50], nimmt die allgemeine körperliche Leistungsfähigkeit weiter $a b$, was im Sinne des Teufelskreises zu einer Zunahme der Dyspnoe führt. Ob dieser Teufelskreis für COPD-Patienten zutreffend ist, muss jedoch noch genauer untersucht werden. Zusätzlich kann dies einen kontinuierlichen Rückzug aus sämtlichen Freizeit- und Alltagsaktivitäten auslösen, was mit einem Verlust von positiven Situationen sowie sozialer Isolation einhergeht und somit Depressivität begünstigt.
Die „Angst vor Dyspnoe“ kann zu einem negativen emotionalen Zustand führen, der die Bereitschaft zur Wahrnehmung atemnotbezogener Symptome erhöht, was Panikattacken auslösen kann [20]. Zudem sind COPD-Patienten, die lebensbedrohliche Atemnotzustände erleben, enormem Stressempfinden ausgesetzt [33, 51]. Eine erhöhte Sensitivität für Atemveränderungen kann wiederum zu einer Vermeidung von Aktivitäten führen, die Atemnot hervorrufen. Somit entsteht ein Teufelskreis, der durch eine $\mathrm{Zu}$ nahme der Dyspnoe, einer Verschlechterung der körperlichen Leistungsfähigkeit und genereller Vermeidung körperlicher Aktivität geprägt ist.

Bezüglich der Angst vor körperlichen Symptomen (BSQ) [43] zeigte sich ein Unterschied zwischen den verglichenen Gruppen. Patienten mit auffälligen Depressivitätswerten verfügen über stärkere Angst vor körperlichen Symptomen als Patienten mit unauffälligen Werten. Dies stellt einen wichtigen Befund dar, da diese Tatsache dafür sprechen könnte, dass COPD-Patienten mit auffälligen Depressivitätswerten generell sensibler bezüglich der Einschätzung ihrer körperlichen Symptome sind.

Überraschend ist, dass sich die untersuchten Patientengruppen nicht in den objektiven medizinischen Parametern unterscheiden. Patienten mit auffälligen Depressivitätswerten schneiden in der Lungenfunktion und dem 6-Minuten-Gehtest nicht schlechter ab. Dieses Ergebnis steht zum Teil im Widerspruch zu vorherigen Studien, bei denen Depressivität mit einer verminderten körperlichen Leistungsfähigkeit und geringerer Gehstrecke in Metern einhergeht [27,40,41]. Laut Kim et al. [27] scheint es jedoch der Fall zu sein, dass Depressivität nicht die objektive Schwere der COPD beeinflusst, sondern die subjektiv vom Patienten empfundene eingeschränkte Funktionalität. Das Ergebnis ist zusätzlich eventuell auf den hohen Anteil von COPD-Patienten in Stadium IV $(72,9 \%)$ mit einer stark beeinträchtigten Lungenfunktion zurückzuführen, so dass gegebenenfalls in diesem Extrembereich der Zusammenhang zwischen objektiv messbarer Krankheitsschwere und Depressivität nicht vorliegt.

Eine Limitierung der Studienergebnisse stellt die selektive Stichprobe dar. Die Daten wurden an einer klinischen Patientengruppe mit COPD-Schweregrad III und IV erhoben. Außerdem sind die Untersuchten im Vergleich zu epidemiologischen Studien relativ jungen Alters und das weibliche Geschlecht überwiegt leicht (57,3\%). Zudem engt die Einschränkung auf eine einzige klinische Einrichtung die Repräsentativität ein. Inwiefern sich die Ergebnisse auf andere Kliniken und ambulante Patienten mit den Schweregraden I und II übertragen lassen, bleibt zu überprüfen. Aufgrund des Querschnittdesigns ist es nicht möglich, Aussagen über den Verlauf von COPD-spezifischen Ängsten zu treffen. Es ist denkbar, dass ein engmaschigeres Längsschnittdesign die Krankheitsängste in den verschiedenen Stadien besser abbilden könnten, da sich die Ängste von dem Zeitpunkt der Diagnosestellung bis zum Terminalstadium verändern können [52]. Kritisch zu betrachten ist weiterhin die Einteilung der Untersuchten in eine Gruppe von Patienten mit auffälligen Depressivitätswerten und eine Gruppe mit unauffälligen Depressivitätswerten mittels eines einzigen Screeninginstrumentes, der Hospital Anxiety and Depression Scale [42]. In zukünftigen Studien könnten in einem zweiten Schritt zusätzliche kategoriale Instrumente zur Abklärung der psychischen Symptomatik eingesetzt werden. Die HADS hat sich jedoch zur Erhebung in Studien mit körperlich Erkrankten als nützlich erwiesen, lediglich der empfohlene Cut-offWert schwankt $[53,54]$. Zusätzlich ist es ein nützliches Verfahren zur Erfassung der Depressivität bei körperlichen Erkrankungen, da es gänzlich auf körperliche Indikatoren psychischer Belastung 
verzichtet. Darüber hinaus wäre es wichtig zu klären, ab welchem Ausmaß krankheitsbezogene Ängste als dysfunktional betrachtet werden müssen. Angst kann sich im Sinne eines Copingstils, durch den Patienten rechtzeitig ärztliche Unterstützung suchen oder Lebensstilveränderungen, wie die Entscheidung zum Nichtrauchen, prognostisch günstig auswirken [55].

Zusammenfassend lässt sich feststellen, dass die vorliegende Studie die hohe Relevanz der Erfassung des Konstruktes krankheitsspezifischer Ängste bei COPD aufzeigt. Die Relevanz COPD-bezogener Ängste für den Krankheitsverlauf hat nicht nur Augenscheinvalidität, sondern zeigt sich auch im Zusammenhang mit Depressivität. Patienten mit auffälligen Depressivitätswerten leiden massiv unter Ängsten bezüglich der COPD-spezifischen Symptomatik und zusätzlichen Folgen dieser chronisch verlaufenden und potenziell lebensbedrohlichen Erkrankung. Die rechtzeitige Identifikation und angemessene Behandlung von krankheitsspezifischen Ängsten und Depressivität hat offensichtlich eine große Bedeutung für den Verlauf von COPD. Ein Screening psychischer Belastungen zu Beginn der Behandlung scheint ratsam, um eine frühe Identifikation belasteter Patienten zu gewährleisten und eine notwendige Basis für entsprechende Indikationsentscheidungen zu haben.

\section{Danksagung}

Wir danken der Firma Boehringer Ingelheim Pharma GmbH \& Co. KG für die freundliche Unterstützung dieser Studie.

\section{Interessenkonflikt}

\section{$\nabla$}

Die Firma Boehringer unterstützte die Durchführung dieser Studie durch Finanzmittel für ein Promotionsstipendium an das Klinikum Berchtesgadener Land. Diese Zuwendung hatte keinen Einfluss auf die im vorliegenden Artikel vorgestellten Ergebnisse.

\section{Literatur}

1 WHO. World Health Statistics 2008. Geneva: 2008

2 Buist AS, McBurnie MA, Vollmer WM et al. International variation in the prevalence of COPD (the BOLD Study): a population-based prevalence study. Lancet 2007; 370: $741-750$

3 The Asia Pacific COPD Roundtable Group. Global Initiative for Chronic Obstructive Lung Disease strategy for the diagnosis, management and prevention of chronic obstructive pulmonary disease: an Asia-Pacific perspective. Respirology 2005; 10: 9-17

4 Rutten-van Molken MP, Oostenbrink JB, Tashkin DP et al. Does quality of life of COPD patients as measured by the generic EuroQol five-dimension questionnaire differentiate between COPD severity stages? Chest 2006; 130: 1117 - 1128

5 Hill $K$, Geist R, Goldstein RS et al. Anxiety and depression in end-stage COPD. Eur Respir J 2008; 31: 667-677

6 Maurer J, Rebbapragada $V$, Borson $S$ et al. Anxiety and depression in COPD: current understanding, unanswered questions, and research needs. Chest 2008; 134: 43-56

7 Di Marco F, Verga M, Reggente $M$ et al. Anxiety and depression in COPD patients: The roles of gender and disease severity. Respir Med 2006; 100: $1767-1774$

8 van Manen JG, Bindels PJ, Dekker FW et al. The influence of COPD on health-related quality of life independent of the influence of comorbidity. J Clin Epidemiol 2003; 56: 1177-1184

9 Kühl K, Schürmann W, Rief W. Mental disorders and quality of life in COPD patients and their spouses. Int J Chron Obstruct Pulmon Dis 2008; 3: 727-736

10 Reuter K, Härter M. Diagnostik psychischer Belastungen und Störungen bei körperlichen Erkrankungen. In: Härter M, Baumeister H, Bengel J
Hrsg. Psychische Störungen bei körperlichen Erkrankungen. Berlin: Springer; 2007: 15-28

11 van Manen JG, Bindels PJ, Dekker FW et al. Risk of depression in patients with chronic obstructive pulmonary disease and its determinants. Thorax 2002; 57: 412-416

12 WHO. International Classification of Diseases (10th revision). Genf: 1992

13 Eifert GH. Cardiophobia: a paradigmatic behavioural model of heart-focused anxiety and non-anginal chest pain. Behav Res Ther 1992; 30 : $329-345$

14 Yohannes AM, Baldwin RC, Connolly MJ. Depression and anxiety in elderly outpatients with chronic obstructive pulmonary disease: prevalence, and validation of the BASDEC screening questionnaire. Int J Geriatr Psychiatry 2000; 15: 1090-1096

15 Härter MC. Mental illness and physical disorders. Psychother Psychosom Med Psychol 2000; 50: 274-286

16 Katon W, Lin EH, Kroenke K. The association of depression and anxiety with medical symptom burden in patients with chronic medical illness. Gen Hosp Psychiatry 2007; 29: 147-155

17 Herschbach P, Keller M, Knight L et al. Psychological problems of cancer patients: a cancer distress screening with a cancer-specific questionnaire. Br J Cancer 2004; 91: 504-511

18 Dankert A, Duran G, Engst-Hastreiter $U$ et al. Progredienzangst bei Patienten mit Tumorerkrankungen, Diabetes mellitus und entzündlichen Erkrankungen des Bewegungsapperatestischen Erkrankungen. Rehabilitation 2003; 42: 155-163

19 Kühl K, Kuhn C, Kenn Ket al. [The COPD-Anxiety-Questionnaire (CAF): a new instrument to assess illness specific anxiety in COPD patients] Psychother Psychosom Med Psychol 2011; 61: e1-9

20 Livermore N, Butler JE, Sharpe L et al. Panic attacks and perception of inspiratory resistive loads in chronic obstructive pulmonary disease. Am J Respir Crit Care Med 2008; 178: 7-12

21 Livermore N, Sharpe L, McKenzie D. Panic attacks and panic disorder in chronic obstructive pulmonary disease: a cognitive behavioral perspective. Respir Med 2010; 104: 1246-1253

22 Borak J, Sliwinski P, Piasecki $Z$ et al. Psychological status of COPD patients on long term oxygen therapy. Eur Respir J 1991; 4: 59-62

23 Engström CP, Persson LO, Larsson $S$ et al. Functional status and well being in chronic obstructive pulmonary disease with regard to clinical parameters and smoking: a descriptive and comparative study. Thorax 1996; 51: $825-830$

24 Eisner MD, Blanc PD, Yelin EH et al. Influence of anxiety on health outcomes in COPD. Thorax 2010; 65: 229-234

25 Aydin IO, Ulusahin A. Depression, anxiety comorbidity, and disability in tuberculosis and chronic obstructive pulmonary disease patients: applicability of GHQ-12. Gen Hosp Psychiatry 2001; 23: 77-83

26 Simon NM, Weiss AM, Kradin $R$ et al. The relationship of anxiety disorders, anxiety sensitivity and pulmonary dysfunction with dyspnearelated distress and avoidance. J Nerv Ment Dis 2006; 194: 951 -957

27 Kim HF, Kunik ME, Molinari VA et al. Functional impairment in COPD patients: the impact of anxiety and depression. Psychosomatics 2000; 41: $465-471$

28 Gudmundsson G, Gislason T, Janson C et al. Risk factors for rehospitalisation in COPD: role of health status, anxiety and depression. Eur Respir J 2005; 26: 414-419

29 Brenes GA. Anxiety and chronic obstructive pulmonary disease: prevalence, impact, and treatment. Psychosom Med 2003; 65: 963-970

30 Vögele $C$, von Leupoldt A. Mental disorders in chronic obstructive pulmonary disease (COPD). Respir Med 2008; 102: 764- 773

31 Hynninen KM, Breitve $M H$, Wiborg AB et al. Psychological characteristics of patients with chronic obstructive pulmonary disease: a review. J Psychosom Res 2005; 59: 429-443

32 Eke M, McNally RJ. Anxiety sensitivity, suffocation fear, trait anxiety, and breath-holding duration as predictors of response to carbon dioxide challenge. Behav Res Ther 1996; 34: 603-607

33 McCathie HC, Spence SH, Tate RL. Adjustment to chronic obstructive pulmonary disease: the importance of psychological factors. Eur Respir J 2002; 19: 47-53

34 Barnett M. Chronic obstructive pulmonary disease: a phenomenological study of patients' experiences. J Clin Nurs 2005; 14: 805-812

35 Kanervisto M, Paavilainen E, Astedt-Kurki P. Impact of chronic obstructive pulmonary disease on family functioning. Heart Lung 2003; 32: $360-367$

36 Kanervisto $M$, Paavilainen E, Heikkila J. Family dynamics in families of severe COPD patients. J Clin Nurs 2007; 16: 1498-1505 
37 Mikkelsen RL, Middelboe T, Pisinger C et al. Anxiety and depression in patients with chronic obstructive pulmonary disease (COPD). A review. Nord J Psychiatry 2004; 58: 65 - 70

$38 \mathrm{Ng}$ TP, Niti M, Tan WC et al. Depressive symptoms and chronic obstructive pulmonary disease: effect on mortality, hospital readmission, symptom burden, functional status, and quality of life. Arch Intern Med 2007; 167: 60-67

39 Garrod R, Marshall J, Barley E et al. Predictors of success and failure in pulmonary rehabilitation. Eur Respir J 2006; 27: 788 - 794

40 Yeh ML, Chen HH, Liao YC et al. Testing the functional status model in patients with chronic obstructive pulmonary disease. J Adv Nurs 2004; 48: $342-350$

41 Felker B, Katon $W$, Hedrick SC et al. The association between depressive symptoms and health status in patients with chronic pulmonary disease. Gen Hosp Psychiatry 2001; 23: 56-61

42 Snaith RP, Zigmond AS. The hospital anxiety and depression scale. $\mathrm{Br}$ Med J (Clin Res Ed) 1986; 292: 344

43 Chambless DL, Caputo GC, Bright $P$ et al. Assessment of fear of fear in agoraphobics: the body sensations questionnaire and the agoraphobic cognitions questionnaire. J Consult Clin Psychol 1984; 52: 1090-1097

44 Hien $P$, Morr $H$. Six-minute walk test in chronic lung and heart disease technical aspects, interpretation, limitations. Pneumologie 2002; 56: $558-566$

45 Berg P, Book K, Dinkel A et al. Fear of progression in chronic diseases. Psychother Psychosom Med Psychol 2011; 61: 32 - 37

46 Uhlemann C. Wie die Lunge die Lebenskraft bestimmt. Forsch Komplementärmed 2007; 14: 173-179
47 Vogelmeier C, Buhl R, Criée CP et al. Guidelines for the diagnosis and therapy of COPD issued by Deutsche Atemwegsliga und Deutsche Gesellschaft für Pneumologie und Beatmungsmedizin. Pneumologie 2007; 61: e1 - 40

48 Worth H, Meyer A, Folgering $H$ et al. Recommendations of the German Respiratory League on sports and physical training for patients with obstructive respiratory tract diseases. Pneumologie 2000; 54: 61 -67

49 Leeuw M, Goossens ME, Linton SJ et al. The fear-avoidance model of musculoskeletal pain: current state of scientific evidence. J Behav Med 2007; 30: 77-94

50 Vlaeyen JW, Linton SJ. Fear-avoidance and its consequences in chronic musculoskeletal pain: a state of the art. Pain 2000; 85: 317-332

51 Andenaes $R$, Kalfoss $M H$, Wahl AK. Coping and psychological distress in hospitalized patients with chronic obstructive pulmonary disease. Heart Lung 2006; 35: 46-57

52 Engst-Hastreiter $U$, Duran G, Henrich $G$ et al. Progredienzangst (PA) bei chronischen Erkrankungen (Rheumatischen Erkrankungen, Krebserkrankungen und Diabetes mellitus). Akt Rheumatol 2004; 29: 83-91

53 Singer S, Kuhnt S, Gotze $\mathrm{H}$ et al. Hospital anxiety and depression scale cutoff scores for cancer patients in acute care. Br J Cancer 2009; 100: 908-912

54 Olsson I, Mykletun A, Dahl AA. The Hospital Anxiety and Depression Rating Scale: a cross-sectional study of psychometrics and case finding abilities in general practice. BMC Psychiatry 2005; 5: 46

55 Herrmann C, Brand-Driehorst S, Buss $U$ et al. Effects of anxiety and depression on 5-year mortality in 5,057 patients referred for exercise testing. J Psychosom Res 2000; 48: 455-462 